\title{
Pierre Robin sequence: Subdivision, data, theories, and treatment - Part 4: Recommended management and treatment of Pierre Robin sequence and its application
}

\begin{tabular}{|l|}
\hline Access this article online \\
\hline Website: \\
www.amsjournal.com \\
\hline DOI: \\
10.4103/2231-0746.186136 \\
\hline Quick Response Code: \\
\hline
\end{tabular}

\author{
Kurt-W Bütow ${ }^{1,2,3}$, Sharan Naidoo ${ }^{1}$, Roger Arthur Zwahlen ${ }^{4}$, Jean A. Morkel ${ }^{5}$ \\ ${ }^{1}$ Department of Maxillofacial and Oral Surgery, Facial Cleft Deformity Clinic, University of Pretoria, ${ }^{2}$ Suite \\ A2-Maxillofacial Surgery, The Wilgers Hospital, Pretoria, ${ }^{3}$ Department of Maxillofacial Surgery, \\ Division of Dentistry, College of Health Sciences, University of KwaZulu-Natal, Durban, \\ ${ }^{5}$ Department Maxillofacial and Oral Surgery, University of the Western Cape, Cape Town, South Africa, \\ ${ }^{4}$ Discipline of Oral and Maxillofacial Surgery, Faculty of \\ Dentistry, University of Hong Kong, Hong Kong SAR, PR China
}

Address for correspondence: Prof. Roger Arthur Zwahlen, Oral and Maxillofacial Surgery, Faculty of Dentistry, The University of Hong Kong, 34 Hospital Road, Hong Kong SAR, PR China.

E-mail: zwahlen@hku.hk

\section{ABSTRACT}

Context: The disorder currently accepted as Pierre Robin syndrome/anomaly/sequence (PRS) has been plagued by controversy ever since initially being described. Controversy exists not only about the appropriate terminology and etiopathogenesis of the disorder but also about its management. Therefore, clinical findings and treatment outcomes of a large database of 266 PRS cases were compared with the current state of knowledge in the scientific literature related to history, clinical description, diagnostic criteria, epidemiology, theories of oligohydramnios, mandibular catch-up growth, midfacial hyperplasia, and early management. Aims of Part 4: To provide a systematic treatment protocol for Fairbairn-Robin triad (FRT) and Siebold Robin sequence (SRS) patients based on clinical findings and experience with 266 PRS cases. Subjects and Methods: A plethora of treatment modalities and their outcome in literature have been compared to those applied in this database and their outcomes. Results: The management of SRS/FRT depends on various factors including compromised airways, feeding difficulties, as well as the sequence of the reconstructive ladder. Conclusion: Based on the novel PRS subdivisions, a stepwise sequential treatment approach is outlined, addressing the particular needs of each disorder systematically.

Keywords: Fairbairn-Robin triad, management, Pierre Robin sequence, Siebold Robin sequence, treatment

\section{INTRODUCTION}

This final part of the publication series discusses treatment strategies and modalities for patients suffering from Siebold-Robin sequence (SRS) and Fairbairn-Robin triad (FRT). Different clinical findings in these conditions lead to various custom-made treatments. Clear clinical distinctions have been elaborated between SRS and FRT in a total of 266 cases in a database of 4158 recorded cleft cases.

The number of SRS cases reported by a European clinic greatly contributes to the total number of cases reported in the literature. ${ }^{[1]}$ In the database presented here, only a rather small group of cases has been detected and recorded. In many SRS cases, the infants might not have been referred to a cleft clinic seeing that they did not present with a palatal cleft, and may most probably have been successfully managed by pediatricians with long-term nasopharyngeal intubation treatment.

This is an open access article distributed under the terms of the Creative Commons Attribution-NonCommercial-ShareAlike 3.0 License, which allows others to remix, tweak, and build upon the work non-commercially, as long as the author is credited and the new creations are licensed under the identical terms.

For reprints contact: reprints@medknow.com

Cite this article as: Bütow KW, Naidoo S, Zwahlen RA, Morkel JA. Pierre Robin sequence: Subdivision, data, theories, and treatment - Part 4: Recommended management and treatment of Pierre Robin sequence and its application. Ann Maxillofac Surg 2016;6:44-9. 
This analysis is based on 266 Pierre Robin syndrome (PRS) cases which represent a part of an overall database of 4158 cleft cases recorded at the largest cleft lip and palate unit in South Africa.

\section{SUBJECTS AND METHODS}

The management of PRS patients should address four problems:

- Compromised or obstructed airways

- Feeding difficulties

- Timing and sequence of primary palatal reconstruction (FRT) as well as the treatment of mandibular micro-/retrognathic deformity

- Any secondary treatment in SRS/FRT patients.

\section{Airways}

Not all SRS/FRT cases necessarily present with compromised airways due to glossoptosis. However, in FRT cases with wide U-shaped palatal clefts, the tongue may slot into, thus being displaced into the nasal cavity, causing airway obstruction.

Less severe airway impairments in SRS and FRT cases are characterized by:

- Coexisting ankyloglossia

- Midfacial hyperplasia

- Other unknown factors associated with pharyngeal and tracheal development.

Treatment strategies depend on the severity of compromised airways and may encompass:

- Application of a palatal plate with a pharyngeal extension (SRS) raising the soft palate (sP)

- Palatal obturator with dorsal extension (FRT) [Figure 1]

- Nasopharyngeal intubation

- Glossopexy

- Mandibular distraction osteogenesis

- Tracheotomy

- Timely and sequential palatal reconstruction (FRT) [Tables 1 and 2].

\section{Feeding}

In this study, the patients who underwent palatal plate therapy were evaluated [Table 3]. Some patients could not tolerate an extended plate during feeding due to breathing difficulties. In a few patients suffering from a severe gastroesophageal reflux, primary reconstruction of the cleft palate was not feasible before laparoscopic Nissen fundoplication surgery had been performed.

A few cases developed premature exhaustion during feeding due to lower facial third deformities such as micrognathia, microglossia, and glossoptosis that caused a reduced fluid intake. In one case, a percutaneous endoscopic gastrostomy (PEG) became necessary to avoid dehydration [Figure 2].

\section{Primary reconstruction of the palate}

At the Facial Cleft Deformity Clinic in the University of Pretoria, Republic of South Africa, the sequence of palatal reconstruction was changed three times from 1983 to 2015, as follows:

- Hard and soft palate (hPsP) cleft closure in a single intervention. In cases with only a sP cleft plus a severe micrognathia, the palate was reconstructed in a single procedure at 12 months of age

- Hard palate $(\mathrm{hP})$ reconstruction at 6 months, followed by sP closure at 12 months of age

- $\mathrm{sP}$ closure at 7 months, followed by hP reconstruction 11 months later ${ }^{[2]}[$ Table 4].

Most patients were operated according to the third protocol, apart from those born with a sP cleft only.

\section{Secondary reconstruction in Siebold-Robin sequence and Fairbairn-Robin triad patients}

For FRT patients, most secondary reconstructions due to velopharyngeal incompetency consisted of velopharyngeal flap procedures. In a few severe cases, dynamic sphincteroplasty or palatal distraction was performed. Generally, it prevails that the wider the cleft and the shorter the sP, the more likely velopharyngeal incompetency will occur. A velopharyngeal incompetency is further amplified in the presence of hypoplastic adenoidal tissue. Furthermore, nonoperated sP halves are often asymmetrical in length possibly contributing to a unilateral velopharyngeal insufficiency.

Not all cases displayed sufficient catch-up growth in adulthood resulting in persistent mandibular hypoplasia. After orthodontic

\begin{tabular}{lcccc}
\hline Table 1: Compromised airways & & \\
\hline Total PRS & SRS & \multicolumn{2}{c}{ FRT } \\
\hline 266 & 21 & \multicolumn{2}{c}{245} \\
Severely compromised & \multicolumn{2}{c}{$\begin{array}{c}95 \\
\text { airways }\end{array}$} & \multicolumn{4}{c}{ Numbers } & Successful & Numbers & Successful \\
Treatments & 2 & 0 & 187 & 159 \\
Palatal plate therapy & 0 & - & 16 & 14 \\
Nasopharyngeal intubation & 0 & - & 13 & 3 \\
Glossopexy & 6 & 4 & 3 & 2 \\
Mandibular distraction & 2 & 2 & 7 & 7 \\
Tracheostomy & 2 &
\end{tabular}

PRS = Pierre Robin syndrome; SRS = Siebold-Robin sequence; FRT $=$ Fairbairn-Robin triad

\section{Table 2: Treatment protocol for SRS and FRT}

\begin{tabular}{|c|c|c|}
\hline & SRS & FRT \\
\hline \multicolumn{3}{|l|}{ Main problems } \\
\hline Airway & Yes & Yes \\
\hline Feeding & Sometimes & Yes \\
\hline Oro-/nasotracheal intubation & $\begin{array}{l}\text { (for tracheostomy/ } \\
\text { distraction) }\end{array}$ & $\begin{array}{l}\text { Yes (cleft } \\
\text { surgery) }\end{array}$ \\
\hline \multicolumn{3}{|l|}{ Airway treatment (postnatal sequence) } \\
\hline Intubation if necessary (ventilation) & Yes & $\begin{array}{l}\text { Yes (immediately, } \\
\text { or during following } \\
3 \text { weeks, if airway } \\
\text { situation worsens) }\end{array}$ \\
\hline Plate with distal extension & $\begin{array}{l}\text { Seldom (= extraoral } \\
\text { fixation) }\end{array}$ & Yes \\
\hline Glossopexy & Seldom & Yes \\
\hline Nasopharyngeal intubation (long-term) & Yes & Yes \\
\hline Tracheostomy* (or first distraction) & Yes & Yes \\
\hline Distraction* (closure of tracheostomy) & Yes & Yes \\
\hline \multicolumn{3}{|l|}{ Feeding treatment (postnatal sequence) } \\
\hline Nasogastric tube & Yes & Yes \\
\hline Plate treatment (as obturator) & No & Yes \\
\hline Gastrostomy & Yes & Yes \\
\hline
\end{tabular}

*Treatments can be applied in a reversed order, depending on the age and circumstances of patient ${ }^{[2]}$ 


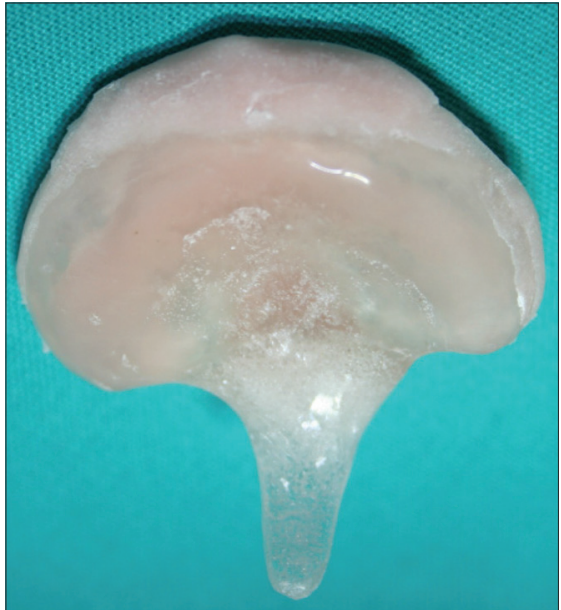

Figure 1: Suction and drinking plate with pharyngeal extension

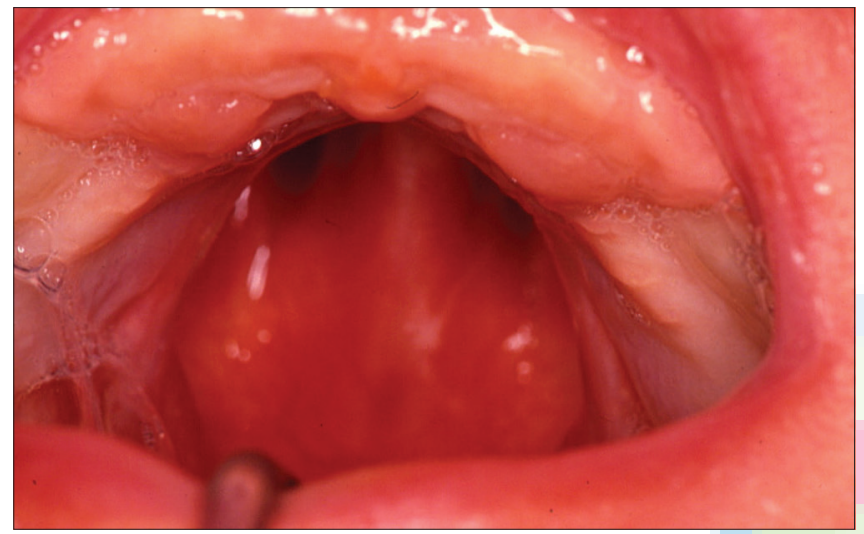

Figure 3: Hard palate width before soft palate repair

\begin{tabular}{|c|c|c|c|c|}
\hline Total PRS & \multicolumn{2}{|c|}{ SRS } & \multicolumn{2}{|c|}{ FRT } \\
\hline 266 & \multirow{2}{*}{\multicolumn{2}{|c|}{$\begin{array}{l}21 \\
16\end{array}$}} & \multicolumn{2}{|c|}{245} \\
\hline Feeding problems & & & \multicolumn{2}{|c|}{103} \\
\hline Treatments & Numbers & Successful & Numbers & Successful \\
\hline Plate therapy & 0 & - & 187 & 143 \\
\hline Reflux-medical treatment & 0 & - & 8 & 1 \\
\hline Nissen & 0 & - & 7 & 6 \\
\hline PEG & 1 & 1 & 15 & 15 \\
\hline
\end{tabular}

PRS = Pierre Robin syndrome; SRS = Siebold-Robin sequence; $F R T=$ Fairbairn-Robin triad

decompensation and alignment, the mandibular retrognathia and lack of chin projection were corrected by means of a bilateral sagittal split osteotomy and/or genioplasty.

The accurate number of patients undergoing secondary reconstruction could not be determined as some of the patients underwent orthognathic surgery at institutions other than the Facial Cleft Deformity Clinic due to geographical and logistic reasons.

\section{RESULTS}

A palatal plate with pharyngeal extension for compromised

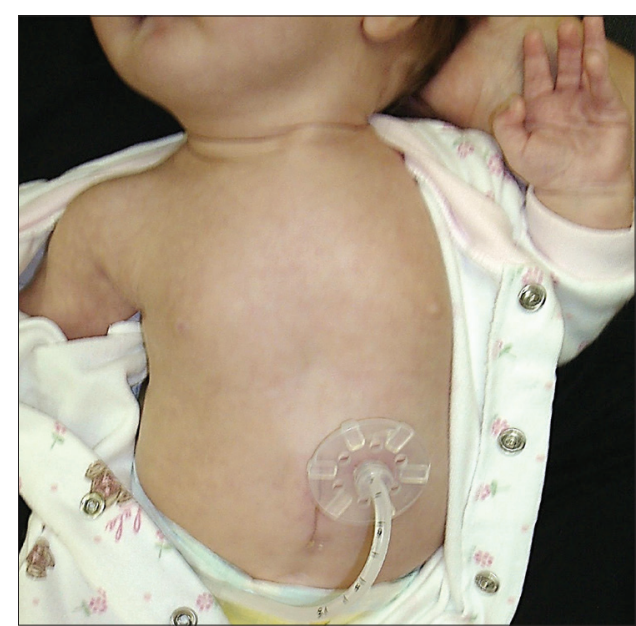

Figure 2: Gastrostomy with percutaneous endoscopic gastrostomy

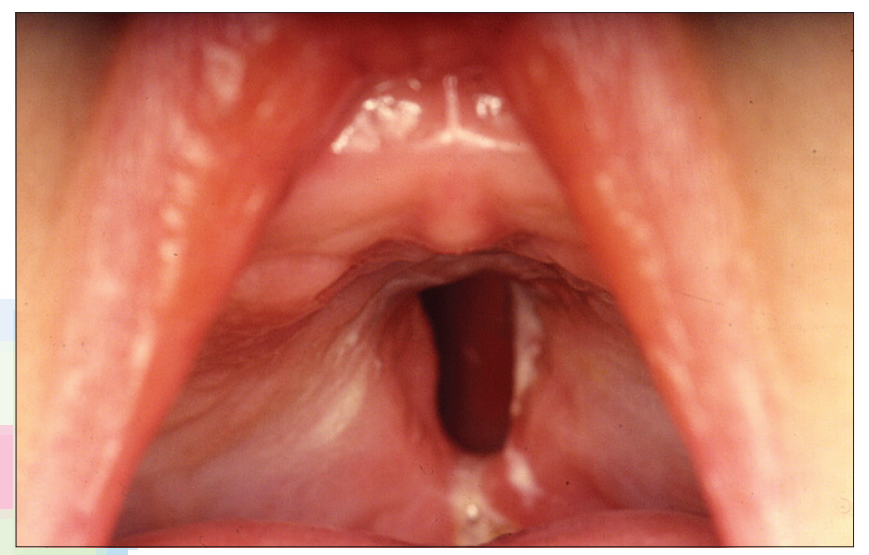

Figure 4: Hard palate width, 11 months after the soft palate was repaired

airways was inserted in two SRS cases. For both patients, the treatment proved unsuccessful.

Of the 187 FRT patients, $19.3 \%$ (36 of 187) presented with ankyloglossia, and consequently with less severe compromised airways. However, 25\% (9 of these 36) presented with severe compromised airways, of which six presented with additional feeding problems.

Of the FRT cases, 159 (84.1\%) could be treated successfully with a palatal obturator plate with pharyngeal extension [Figure 1].

Less commonly used treatment modalities in compromised airways were:

- Long-term nasopharyngeal intubation for a period of up to 3 months in 16 FRT patients with a success rate of $87.5 \%$

- Glossopexy, in terms of a tongue-mandibular adhesion, was performed on 13 FRT patients. Whereas early dehiscence occurred in $76.9 \%$ (10 of the 13 cases), concomitant airway improvement appeared in $80.0 \%$ (8 of the 10 cases). The latter probably happened due to postsurgical scarring processes within the tongue and/or oral floor

- Six (28.6\%) SRS and $7(2.9 \%)$ FRT patients underwent distraction osteogenesis due to compromised airways with a success rate of $66.7 \%$ in both subgroups 
Table 4: Cleft palate reconstruction (Fairbairn-Robin triad)

\begin{tabular}{|c|c|c|c|c|}
\hline Total 245 & SP cleft & hPsP cleft & hPsP cleft & hPsP cleft \\
\hline \multicolumn{5}{|l|}{ Sequence of procedure } \\
\hline $\begin{array}{l}\text { Done elsewhere } \\
\text { Unknown procedure }\end{array}$ & $\begin{array}{l}\text { One procedure * } \\
\text { (sP } 7 \mathrm{~m} \text { or } 12 \mathrm{~m})\end{array}$ & $\begin{array}{l}\text { One procedure * } \\
\text { (hPsP } 12 \mathrm{~m})\end{array}$ & $\begin{array}{l}\text { Two procedures ** } \\
\text { (hP } 6 \mathrm{~m} ; \mathrm{sP} 12 \mathrm{~m})\end{array}$ & $\begin{array}{l}\text { Two procedures *** } \\
(\mathrm{sP} 7 \mathrm{~m} ; \mathrm{hP} 18 \mathrm{~m})\end{array}$ \\
\hline \multicolumn{5}{|l|}{ Number } \\
\hline 12 & 23 & 4 & 21 & 185 \\
\hline
\end{tabular}

*First protocol; ${ }^{* *}$ Second protocol; ***Third protocol

- Immediate postnatal tracheostomy as a last resort to overcome compromised airways was performed on $2(9.5 \%)$ SRS patients and $4(1.6 \%)$ FRT patients. These cases make part of an overall of 7 (2.9\%) FRT patients undergoing tracheostomy to overcome severely compromised airways [Table 1].

In $76.5 \%$ (143 of 187 ) of FRT patients, palatal obturator plate treatment succeeded in overcoming feeding problems. Reflux problems were encountered in eight FRT patients. Only one of the latter could be treated successfully with medication, whereas the remaining seven patients had to undergo laparoscopic Nissen fundoplication surgery which proved successful in six patients.

Patients (SRS 1 [4.8\%] and FRT 15 [6.1\%]) presenting with persistent feeding problems and poor weight gain received a PEG, mainly for a period of 6-12 months [Table 3].

Primary surgery of the soft and hard cleft palate was performed using one of the three previously described treatment protocols. The first is single stage surgical intervention which had previously been used to repair hPsP clefts. In this study population, it was used for rare isolated sP clefts in 23 out of the 245 (9.4\%) FRT patients. The second protocol was implemented as a two-stage surgical procedure, whereby the $\mathrm{hP}$ was reconstructed at six and the sP at 12 months of age. In the reversed third protocol, initially, the sP cleft was repaired at seven followed by the hP cleft at 18 months of age. This modality was used in $185(75.5 \%)$ of the FRT cases [Table 4].

Secondary reconstructions have mainly been performed to manage velopharyngeal incompetency and mandibular hypoplasia. Velopharyngeal reconstruction was performed in $39(15.9 \%)$ of the 245 FRT cases compared to $44(3.0 \%)$ of a total of 1480 patients with other isolated cleft palate. Two SRS patients underwent secondary distraction osteogenesis to correct mandibular hypoplasia in an anterior-posterior dimension.

\section{DISCUSSION}

The management of SRS/FRT patients is multifaceted, depending on the type and extent of concomitant disorders, including:

- Compromised airways, with or without obstruction

- Feeding difficulties

- Sequence of primary palatal cleft repair (FRT) and treatment of eventually severe mandibular hypoplasia

- Other secondary reconstruction at a later stage.

One of the major threats in these patients is compromised airways, with or without obstruction potentially leading to hypoxia due to glossoptosis. Hypoxia may not always be associated with noticeable obstructive episodes. ${ }^{[3-6]}$

Initially, SRS/FRT infants should undergo a clinical examination encompassing, especially their nutritional status and clinical signs of respiratory obstruction, both in cradle and supine position. If the initial examination does not show any evident airway compromise during the nonfeeding period, the examination should be repeated during feeding. ${ }^{[7]}$ Caouette-Laberge et al. ${ }^{[8]}$ included serial blood gas analysis as well as polysomnographic examinations to monitor their patients. However, polysomnographic studies are not routinely conducted at institutions, ${ }^{[9]}$ including the clinic at which this study was conducted.

In cases with respiratory distress, it is advocated to either establish a nasopharyngeal airway ${ }^{[10,11]}$ or to apply a modified nasopharyngeal tube with oxygen nasal prongs to address shortcomings inherent to traditional nasopharyngeal airway management. ${ }^{[12,13]}$

Various surgical procedures have been used to address compromised airways in SRS/FRT patients. Glossopexy, hyomandibulopexy, subperiosteal release of the mouth floor, and tracheotomy are all known as valuable options to secure

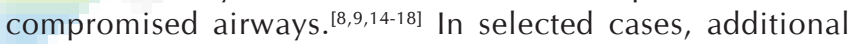
mandibular distraction osteogenesis ${ }^{[19,20]}$ or mandibular forward traction by means of extraoral extended wires ${ }^{[21]}$ was employed.

The glossopexy procedure, with its numerous modifications, such as tongue-lip adhesion ${ }^{[22]}$ or tongue-mandibular adhesion ${ }^{[2]}$ holds several complications such as wound dehiscence, tongue lacerations, salivary ducts injuries, and ankyloglossia secondary to scar tissue formation. ${ }^{[17]}$ In 1977, Randal[ ${ }^{[18]}$ described a modification of the tongue-lip adhesion technique to improve airways. Evaluation of the glossopexy in those 13 cases presented in Table 1 concluded that it served only for temporary airway management. Effective adhesion occurred in only $23.1 \%$ of these cases, suggesting that this procedure should only be considered in cases with minimal feeding disorders.

Very few patients, $28.6 \%$ of SRS and $2.9 \%$ of FRT cases, needed mandibular distraction osteogenesis. Mandibular distraction osteogenesis has been reported to be useful to prevent tracheostomy procedures in PRS cases. ${ }^{[22]}$ Even though this treatment modality is not suitable in infants with tracheomalacia, it might replace tracheostomy procedures in SRS/FRT patients with severely compromised airways in the future. Prominent lifelong soft tissue scars on the height of the mandibular ramus or angle as well as eventually displaced dental follicles display the most common adverse events of this technique. ${ }^{[23]}$ Mandibular distraction osteogenesis proved successful in addressing airway 
obstruction in $66.7 \%$ of the tracheostomy-dependent children in this study.

Feeding disorders in these PRS children might be subject to oroesophageal motor disorders, motor dysfunction, and/or brainstem dysfunction. ${ }^{[3-6]}$ Feeding difficulties may also occur due to secondary respiratory disorders or chronic airway obstruction. ${ }^{[24]}$ These complications have been split into separate categories, determined by the extent of compromised airways related to oxygen saturation and scale of feeding disorders based on weight gain. ${ }^{[7]}$

Of special concern are neonates with clear signs of respiratory obstruction that worsens in supine position and during feeding. ${ }^{[25]}$ When infants fail to thrive, it is often due to either feeding disorders or respiratory obstruction with subsequent pulmonary hypertension secondary to chronic hypoxia. ${ }^{[25]}$ However, it has to be kept in mind that in syndromic SRS/FRT patients, the underlying syndrome itself might be the main cause for failed thriving. ${ }^{[3]}$

In this database, slightly more severe feeding disorders $(44.7 \%)$ were observed than severely compromised airways (41.4\%). In $76.5 \%$ of FRT patients, the insertion of a pharyngeal extended palatal plate/obturator secured the airways and successfully facilitated feeding. Persistent gastroesophageal reflux was treated with laparoscopic Nissen fundoplication surgery, whereas PEG was performed in cases still reluctant to thrive. Pharyngeal extended palatal obturator therapy in FRT patients and PEG in SRS/FRT patients with continuous feeding problems thus represented the most common treatment strategies in this study. Nasogastric tube feeding seldom represents a long-term solution. It may be used in SRS/FRT patients, on a short-term basis combined with palatal plate therapy (FRT). Therefore, the recommended treatment strategies for SRS and FRT patients vary slightly.

Little has been published relating to cleft palate repair sequencing. It is well understood, that FRT patients may display significant variations in both cleft length and width, ${ }^{[26]}$ constituting challenges for surgical repair. It has been reported that up to $7.1 \%$ of patients developed compromised airways after palatoplasty. ${ }^{[27]}$

As highlighted in Table 4, three different sequences were used for cleft repair in the here presented patients. With the two-stage approach where the sP cleft was repaired first, postoperative compromised airways occurred in only $2.7 \%$. A two-stage approach with an initial SP repair, therefore keeping the $\mathrm{hP}$ cleft, facilitates postoperative breathing in FRT patients. ${ }^{[2]}$ The tongue's position slowly adapts to an anteroinferior positioning of the palatal shelves which favors respiration and feeding. The hP cleft, which shrinks in width over a period of 11 months [Figures 3 and 4] can then be closed at 18 months of age. ${ }^{[28]}$

Velopharyngeal insufficiency after palatal repair occurred in $15.9 \%$, slightly lower compared to the range from $16 \%$ to $38 \%$ reported by others. ${ }^{[29]}$ All cases presented here (39 of 245) were reconstructed using a superior pedicle velopharyngeal flap. Only $1.6 \%$ (4 of 245) underwent an additional dynamic sphincteroplasty. Two $(0.8 \%)$ FRT patients underwent a secondary palatal distraction for velopharyngeal incompetency at 9 years of age. ${ }^{[30]}$

In $67.9 \%$ of the SRS/FRT cases, no mandibular catch-up growth occurred. Once adolescent, these patients underwent bilateral sagittal split osteotomies with or without advancement genioplasty to address their mandibular hypoplasia in the anterior-posterior dimension.

Based on these findings, taking into consideration difficulties that often occur during oro- and/or nasotracheal intubation, ${ }^{[31]}$ the following sequential treatment protocols can be recommended for SRS and $\mathrm{FRT}^{[2]}$ patients with persisting airway compromise [Table 2]:

- SRS - nasopharyngeal tube for 2-3 months, followed by a mandibular distraction osteogenesis, followed by a tracheostomy, in case the previous particular treatment step was unsuccessful

- FRT - pharyngeal extended palatal obturator therapy extending into the $\mathrm{sP}$ and/or to keep the tongue base position anteriorly, ${ }^{[32]}$ a nasopharyngeal tube for $2-3$ months, ${ }^{[13]}$ a glossopexy, a mandibular distraction osteogenesis, and as a last resort a tracheostomy, in case the previous particular treatment step proved to be unsuccessful.

The following shortcomings of these treatment steps should be remembered:

- Soft tissue dehiscence after glossopexy often occurs, but the majority of patients benefit from this procedure due to scar formation in the floor of the mouth and tongue

- Visible scars due to extraoral approaches for the mandibular distraction osteogenesis. However, intraoral approaches and insertion of distractor devices are technically very demanding in neonates and infants and therefore should never be the first choice in addressing compromised airways. In cases of a tracheostomy performed immediately postnatal, consider to postpone the distraction until 12 months of age.

Reviewing prevailing scientific literature related to treatment approaches in PRS patients one might get the notion that currently rather aggressive ways to secure airways are applied, immediately using second tier surgeries, such as tracheostomy, glossopexy, and mandibular distraction osteogenesis. ${ }^{[33,34]}$ This is totally contrary to the treatment protocols derived from this database.

\section{CONCLUSION}

As each SRS and FRT case may present with a specific bucket list of symptoms and priorities, stipulation of treatment protocols remains challenging. Under certain circumstances, the appropriate strategy may vary considerably, especially related to intraoral defects and facial deformities which need to be individually addressed. By means of a large database and the interrelated clinical experience appropriate treatment protocols were developed and successfully applied in both SRS and FRT cases.

Based on the novel PRS subdivisions, a stepwise sequential treatment approach is outlined, addressing the particular needs of each disorder systematically. 


\section{Acknowledgement}

The authors would like to thank Mrs Jennilee Blom for her invaluable job as research assistant.

\section{Financial support and sponsorship}

Nil.

\section{Conflicts of interest}

There are no conflicts of interest.

\section{REFERENCES}

1. Reinert S, Bacher M, Buchenau W, Arand J, Poets G, Göz G. Treatment of severe Pierre Robin sequence- state of the art. J Craniomaxillofac Surg 2008;36Suppl:S22. [O.086].

2. Bütow KW, Zwahlen RA, editors. Pierre Robin sequence treatment. In: Cleft- Ultimate Treatment. $2^{\text {nd }}$ ed. Durban: Reach Publisher; 2016. p. 337-65.

3. Abadie V, Morisseau-Durand MP, Beyler C, Manach Y, Couly G. Brainstem dysfunction: A possible neuroembryological pathogenesis of isolated Pierre Robin sequence. Eur J Pediatr 2002;161:275-80.

4. Renault F, Flores-Guevara R, Soupre V, Vazquez MP, Baudon JJ. Neurophysiological brainstem investigations in isolated Pierre Robin sequence. Early Hum Dev 2000;58:141-52.

5. Baujat G, Faure C, Zaouche A, Viarme F, Couly G, Abadie V. Oroesophageal motor disorders in Pierre Robin syndrome. J Pediatr Gastroenterol Nutr 2001;32:297-302.

6. Baudon JJ, Renault F, Goutet JM, Flores-Guevara R, Soupre V, Gold F, et al. Motor dysfunction of the upper digestive tract in Pierre Robin sequence as assessed by sucking-swallowing electromyography and esophageal manometry. J Pediatr 2002;140:719-23.

7. Wagener S, Rayatt SS, Tatman AJ, Gornall P, Slator R. Management of infants with Pierre Robin sequence. Cleft Palate Craniofac J 2003;40:180-5.

8. Caouette-Laberge L, Bayet B, Larocque Y. The Pierre Robin sequence: Review of 125 cases and evolution of treatment modalities. Plast Reconstr Surg 1994;93:934-42.

9. van den Elzen AP, Semmekrot BA, Bongers EM, Huygen PL, Marres HA. Diagnosis and treatment of the Pierre Robin sequence: Results of a retrospective clinical study and review of the literature. Eur J Pediatr 2001;160:47-53.

10. Rayatt S, Tattman A, Slator R, Gornall P. Re: Whitaker et al. Effective management of airway in Pierre Robin syndrome. Br J Oral Maxillofac Surg 2004;42:176.

11. Whitaker IS, Koron S, Oliver DW, Jani P. Effective management of the airway in the Pierre Robin syndrome using a modified nasopharyngeal tube and pulse oximetry. Br J Oral Maxillofac Surg 2003;41:272-4.

12. Masters IB, Chang AB, Harris M, O’Neil MC. Modified nasopharyngeal tube for upper airway obstruction. Arch Dis Child 1999;80:186-7.

13. Robin P. A fall of the base of the tongue considered as a new cause of nasopharyngeal respiratory impairment: Pierre Robin sequence, a translation 1923. Plast Reconstr Surg 1994;93:1301-3.

14. St-Hilaire H, Buchbinder D. Maxillofacial pathology and management of Pierre Robin sequence. Otolaryngol Clin North Am 2000;33:1241-56.

15. Denny AD, Amm CA, Schaefer RB. Outcomes of tongue-lip adhesion for neonatal respiratory distress caused by Pierre Robin sequence.
J Craniofac Surg 2004;15:819-23.

16. Kirschner RE, Low DW, Randall P, Bartlett SP, McDonald-McGinn DM, Schultz PJ, et al. Surgical airway management in Pierre Robin sequence: Is there a role for tongue-lip adhesion? Cleft Palate Craniofac J 2003;40:13-8.

17. Douglas B. The treatment of micrognathia associated with obstruction by a plastic procedure. Plast Reconstr Surg 1946;1:300-8.

18. Randall P. The Robin anomalad: Micrognathia and glossoptosis with airway obstruction. In: Converse J, editor. Reconstructive Plastic Surgery. Philadelphia: WB Saunders; 1977. p. 2235-45.

19. Burstein FD, Williams JK. Mandibular distraction osteogenesis in Pierre Robin sequence: Application of a new internal single-stage resorbable device. Plast Reconstr Surg 2005;115:61-7.

20. Breugem C, Paes E, Kon M, Mink van der Molen AB. Bioresorbable distraction device for the treatment of airway problems for infants with Robin sequence. Clin Oral Investig 2012;16:1325-31.

21. Baciliero U, Spanio di Spilimbergo S, Riga M, Padula E. Respiratory distress in Pierre Robin sequence: An experience with mandible traction by wires. Int J Oral Maxillofac Surg 2011;40:464-70.

22. Basart H, Kruisinga FH, Breugem CC, Don Griot JP, Hennekam RC, Van der Horst CM. Will the right Robin patient rise, please? Definitions and criteria during management of Robin sequence patients in the Netherlands and Belgium. J Craniomaxillofac Surg 2015;43:92-6.

23. Arnspiger SA, Felder JM $3^{\text {rd }}$, Wood BC, Rogers GF, Oh AK. Carotid artery dissection and stroke complicating treatment of postmandibular distraction ankylosis: A case report. Cleft Palate Craniofac J 2015;52:373-6.

24. Elliott MA, Studen-Pavlovich DA, Ranalli DN. Prevalence of selected pediatric conditions in children with Pierre Robin sequence. Pediatr Dent 1995;17:106-11

25. du Plessis SM, van den Berg HJ, Bütow KW, Hoogendijk CF. Airway and feeding problems in infants with Fairbairn-Robin triad deformities. Curationis 2013;36:E1-9.

26. Godbout A, Leclerc JE, Arteau-Gauthier I, Leclerc LD. Isolated versus Pierre Robin sequence cleft palates: Are they different? Cleft Palate Craniofac J 2014;51:406-11.

27. Costa MA, Murage KP, Tholpady SS, Flores RL. Airway compromise following palatoplasty in Robin sequence: Improving safety and predictability. Plast Reconstr Surg 2014;134:937e-45e.

28. Bütow KW, Hoogendijk CF, Zwahlen RA. Pierre Robin sequence: Appearances and 25 years of experience with an innovative treatment protocol. J Pediatr Surg 2009;44:2112-8.

29. Patel KB, Sullivan SR, Murthy AS, Marrinan E, Mulliken JB. Speech outcome after palatal repair in nonsyndromic versus syndromic Robin sequence. Plast Reconstr Surg 2012;130:577e-584e.

30. Bütow KW. Palatal distraction an option for velopharyngeal incompetence? J Craniomaxillofac Surg 2002;30Suppl:159. [137].

31. Arteau-Gauthier I, Leclerc JE, Godbout A. Can we predict a difficult intubation in cleft lip/palate patients? J Otolaryngol Head Neck Surg 2011;40:413-9.

32. Bacher M, Sautermeister J, Urschitz MS, Buchenau W, Arand J, Poets CF An oral appliance with velar extension for treatment of obstructive sleep apnea in infants with Pierre Robin sequence. Cleft Palate Craniofac J 2011;48:331-6.

33. Cascone P, Papoff P, Arangio P, Vellone V, Calafati V, Silvestri A. Fast and early mandibular osteodistraction (FEMOD) in severe Pierre Robin Sequence. J Craniomaxillofac Surg 2014;42:1364-70.

34. Collins B, Powitzky R, Robledo C, Rose C, Glade R. Airway management in Pierre Robin sequence: Patterns of practice. Cleft Palate Craniofac J 2014;51:283-9. 